\title{
Roswitha Skare
}

"Unsere Freunde, die Maler." Zum Verhältnis von Text und Bild in Christa Wolfs Sommerstück

Christa Wolf's Sommerstück was published in March 1989, just in time to celebrate the 60th birthday of the author in both East and West Germany. In the years after 1989, different paperback editions followed. Interesting to note is that pictures by Hartwig Hamer were included only in the original edition by Aufbau. Accordingly, I would like to focus in this paper on paratextual elements - using the term coined by Gérard Genette in his work Paratexts: Thresholds of Interpretation - before I turn my attention to the word-image relationship between Christa Wolf's text and Hartwig Hamer's pictures.

Bereits seit den siebziger Jahren hatten Christa Wolf und ihr Mann Gerhard Wolf engen Kontakt zu Künstlern, da beide an dem Miteinander von Literatur und bildender Kunst interessiert sind. Dies kommt in der Zusammenarbeit Christa Wolfs mit Künstlern in der Gestaltung ihrer Bücher zum Ausdruck, ${ }^{1}$ bei Lesungen, ${ }^{2}$ aber auch in Gerhard Wolfs Verlag Janus press, und nicht zuletzt in Ausstellungen ${ }^{3}$ und den dazugehörigen Publikationen. Peter Böthig beschreibt dieses Verhältnis folgendermaßen:

Es geht ihnen [Gerhard und Christa Wolf, R.S.] immer um das "Verstehen" eines Bildes, eines ästhetischen Ansatzes, und um jenen Rest an künstlerischer Energie, der beim Verstehen, also beim Sprache-Werden, offen bleiben muß und so als Provokation in die schriftstellerische Arbeit hineinragt. Die unterschiedlichen ästhetischen Wahrnehmungen von Literatur und bildender Kunst werden einander konfrontiert. (Böthig 1996, 7)

Sommerstück erschien zum 60. Geburtstag Christa Wolfs im März 1989 im

Ostberliner Aufbau-Verlag und als Lizenzausgabe im westdeutschen Luchterhand Literaturverlag. In den folgenden Jahren wurde Sommerstück als Taschenbuch in den unterschiedlichen Serien bei dtv und Luchterhand neu aufgelegt. ${ }^{4} 2001$ erschien Sommerstück gemeinsam mit Was bleibt im Band 10 der von Sonja Hilzinger bei Luchterhand herausgegebenen, kommentierten und mit einem Nachwort versehenen gesammelten Werke; im Januar 2008 als Taschenbuch in einer neuen Serie bei Suhrkamp. 
Nun ist es keineswegs ungewöhnlich, dass die Werke Christa Wolfs bis zur Wiedervereinigung im Oktober 1990 zumeist zeitgleich in Ost und West erschienen und dass in den darauf folgenden Jahren andere Ausgaben - sei es Übersetzungen oder Neuauflagen - folgen. Auffallend für Christa Wolfs Sommerstück ist jedoch, dass lediglich die Originalausgabe des Aufbau-Verlages Grafiken Hartwig Hamers enthält.

Literaturwissenschaftliche Arbeiten zu Sommerstück gehen meines Wissens weder auf die unterschiedlichen Umschläge der verschiedenen Ausgaben ein, noch berücksichtigt man die Grafiken Hamers im Zusammenhang mit der Textarbeit. Dies ist überraschend, da man zumindest für die achtziger Jahre davon ausgehen kann, dass Christa Wolf relativ großen Einfluss auf die Gestaltung ihrer Bücher hatte. Dies betrifft die Wahl der Grafiken, aber auch solche Aspekte wie Umschlaggestaltung und Layout. Dass ihr dabei ästhetische wie auch materielle Anliegen wichtig waren, verdeutlichen Christa Wolfs Briefe an ihre Lektorin beim Aufbau-Verlag Angela Drescher vom 21. November 1988 und vom 14. März 1989, in denen sie sowohl auf Satzspiegel als auch auf die Papierqualität und den Einband von Sommerstück eingeht. $^{5}$

Im Folgenden soll mit Gérard Genettes Begrifflichkeit vom Paratext zunächst auf ausgewählte Elemente in den verschiedenen Ausgaben von Sommerstück eingegangen werden, bevor ich auf das Verhältnis von Christa Wolfs literarischen Text und Hartwig Hamers Grafiken zurückkomme.

In seiner Studie Paratexte. Das Buch vom Beiwerk des Buches (in Französisch bereits 1987 unter dem Titel Seuils) zeigt Genette mit Hilfe zahlreicher Beispiele, welche Rolle Titel, Untertitel, Vorworte, Umschlagtexte, aber auch der Bekanntheitsgrad des Autors, sein Alter und Geschlecht, sowie Preise, Ehredoktorwürden u.ä. für eine Interpretation haben. Genette teilt den Paratext in den Peritext, der - wie Schutzumschlag, Titel, Gattungsangabe, Vor- und Nachwort oder auch verschiedene Motti - relativ fest mit dem Buch verbunden ist, und in den Epitext, der Mitteilungen über das Buch enthält, die in der Regel an einem anderen Ort plaziert sind wie Interviews, Briefwechsel oder Tagebücher. 


\begin{tabular}{|c|c|}
\hline \multicolumn{2}{|c|}{ Paratext } \\
\hline $\boldsymbol{K}$ & $y$ \\
\hline Peritext (innerhalb des Buches) & Epitext (außerhalb des Buches) \\
\hline 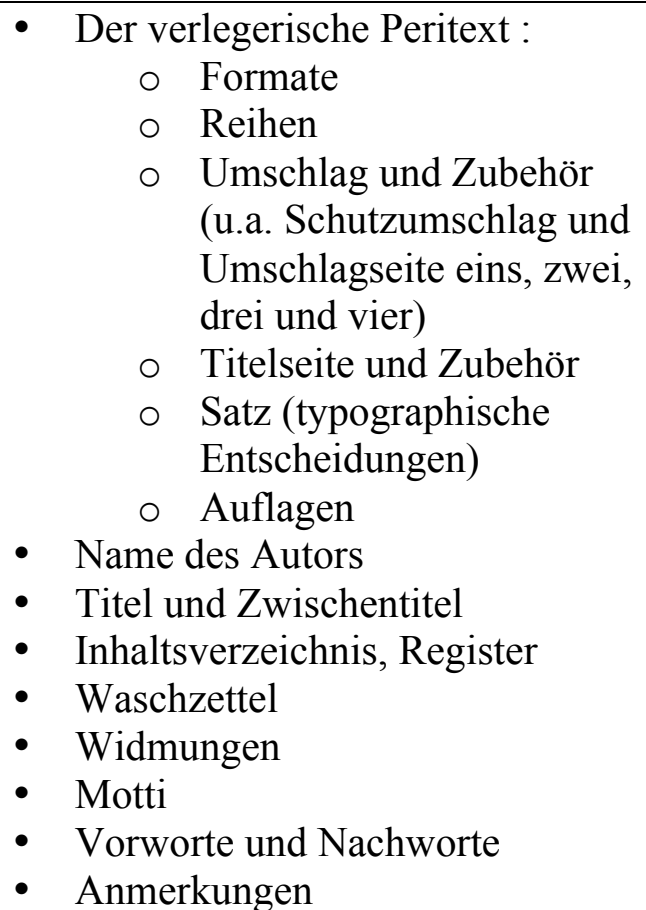 & 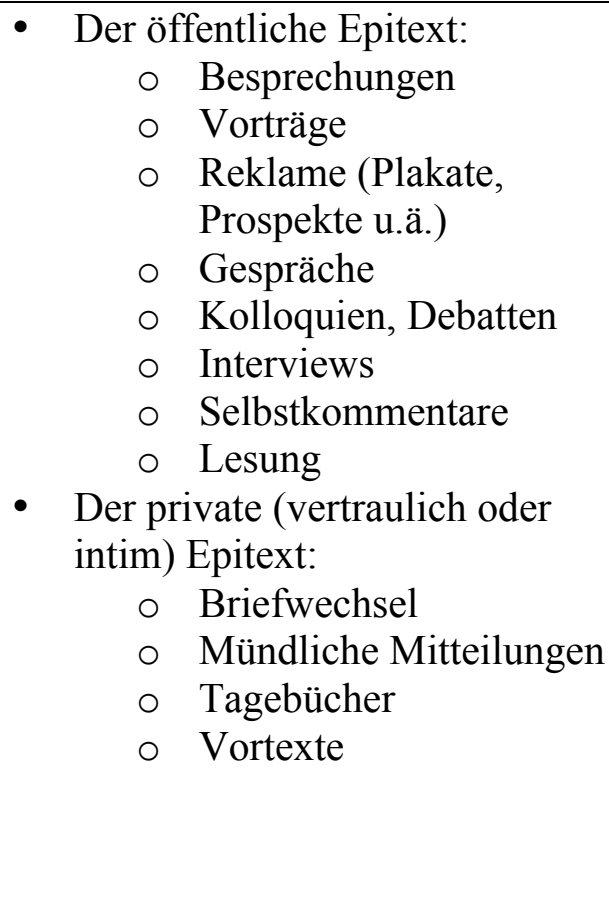 \\
\hline
\end{tabular}

Indem Genette die räumliche Anordnung zum Kriterium dafür macht, ob es sich um peri- bzw. epitextuelle Elemente handelt, ist die Anzahl der jeweiligen Elemente keineswegs stabil, sondern kann sich von Ausgabe zu Ausgabe verändern. Obwohl viele dieser Elemente textbasiert sind, geht Genette auch auf nicht-textliche Elemente wie Format und Umschlaggestaltung ein:

Meistens ist also der Paratext selbst ein Text: Er ist zwar noch nicht der Text, aber bereits Text. Doch muß man zumindest den paratextuellen Wert bedenken, den andere Erscheinungsformen annehmen können: bildliche (Illustrationen), materielle (alles, was zu den typographischen Entscheidungen gehört, die bei der Herstellung eines Buches mitunter sehr bedeutsam sind) oder rein faktische. Als faktisch bezeichne ich einen Paratext, der aus einem Faktum, dessen bloße Existenz, wenn diese der Öffentlichkeit bekannt ist, dem Text irgendeinen Kommentar hinzufügt oder auf seiner Rezeption lastet. (Genette 1989, 14. Hervorh. i. Orig.) 
Obwohl typographische Entscheidungen, das Format eines Buches oder auch die Umschlaggestaltung "im modernen Verlagswesen durch eine wohl unumkehrbare Tendenz zur Standardisierung immer weniger zum Tragen kommen” (ebd., 39), kann die Buchform nicht als selbstverständlich gegeben betrachtet und damit übersehen werden:

When a literary work interrogates the inscription technology that produces it, it mobilizes reflexive loops between its imaginative world and the material apparatus embodying that creation as a physical presence. Not all literary works make this move, of course, but even for those that do not, my claim is that the physical form of the literary artifact always affects what the words (and other semiotic components) mean. (Hayles 2002, 25. Hervorh. i. Orig.)

So steht das größere Format $(14,8$ x 21,7) bei Aufbau offensichtlich im Zusammenhang mit der Größe der reproduzierten Grafiken, ${ }^{6}$ hat aber auch Konsequenzen für die Seitenzahl (191 Seiten) des Buches.

Neben dem unterschiedlichen Format und der damit verbundenen Seitenzahl fällt im Vergleich der beiden Ausgaben aus dem Jahr 1989 - neben den fehlenden Bildern bei Luchterhand - vor allem die unterschiedliche Umschlaggestaltung auf. ${ }^{7}$
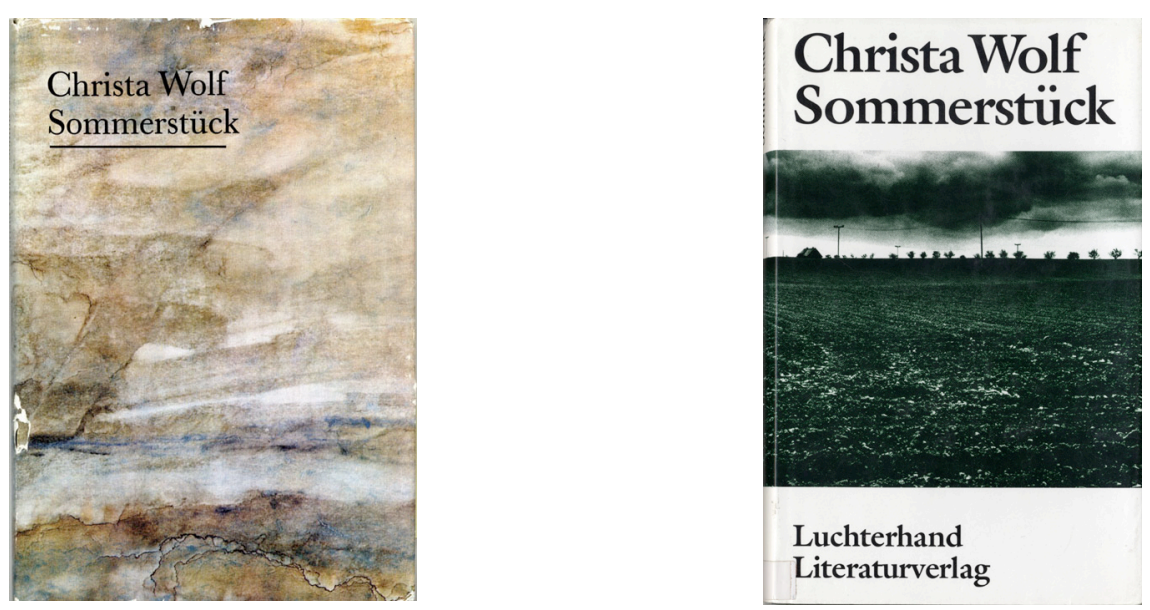

Ein Aquarell Hartwig Hamers wurde vom Aufbau-Verlag sowohl für die Gestaltung des Pappeinbandes als auch des Schutzumschlages verwendet. Dieses Aquarell nimmt den gesamten Umschlag ein; der wenige Text - Name und Titel, sowie ISBN- 
Nummer klein links unten auf der Rückseite - wirken dadurch untergeordnet, heben sich jedoch gleichzeitig durch die Farbwahl vom Hintergrund ab. Der Strich unter dem Titel begrenzt den Text zusätzlich auf diesen linken oberen Teil des Umschlages. Für den Leser des Buches ist es unmöglich zu wissen, inwieweit es sich hier um einen Ausschnitt oder um das gesamte Aquarell handelt und inwieweit die Farbwiedergabe dem Original entspricht, zumal sich die Farbnuancen des Schutzumschlages vom Pappeinband unterscheiden.

In der Luchterhand-Ausgabe nimmt eine schwarz-weiße, mit dunkelgrün unterlegte Landschaftsfotografie den größten Teil des Umschlages ein. Der dunkelgrüne Leineneinband wiederholt dabei die grüne Farbe des Umschlages. Ein weißer Streifen ober- und unterhalb der Fotografie dient als Hintergrund und Kontrast zur schwarzen Schrift: wie in der Aufbau-Ausgabe finden wir hier den Namen Christa Wolfs und den Titel des Buches, jedoch auch den Namen des Verlages, wenn auch in kleineren Buchstaben weniger hervorgehoben. Die Landschaft wirkt menschenleer, lediglich am Horizont sehen wir eine Landstraße mit Bäumen, Stromleitungen und einem Haus, was die Anwesenheit von Menschen andeutet. Durch die eher düsteren Farben und die tief hängenden Wolken am Horizont, weckt dieses Bild Assoziationen an ein bevorstehendes Gewitter. Die Fotografie nimmt zudem nur einen kleinen Teil der Rückseite ein und gibt so Platz für einen Text, der durch Anführungszeichen als Zitat gekennzeichnet ist, jedoch ohne Quellenangabe. Auf dem Klappentext beider Ausgaben wird Sommerstück präsentiert, wobei in der Luchterhand-Ausgabe mehrere Zitate aus dem Text verwendet werden. Der Klappentext als moderne Form des Waschzettels steht in unmittelbarer Nähe zum Text und ist - so Genette - nur einer „eingeschränkten Schicht des Publikums zugänglich, die die Buchhandlungen aufsucht und die Umschläge betrachtet" (Genette 1989, 109). Insgesamt kann man davon ausgehen, dass potentielle Leser/Käufer sich mit Hilfe des Klappentextes einen ersten Eindruck vom Inhalt des Buches verschaffen.

Obwohl der Kauf eines Buches sicherlich nicht allein von dessen Umschlag abhängt, kann man trotzdem annehmen, dass die unterschiedlichen Umschläge beim Betrachter/Leser unterschiedliche Assoziationen und Erwartungen auslösen. Da Hamers Aquarell ohne Titelangabe verwendet wird, ist der Leser relativ frei in seiner Interpretation. Inwieweit der Leser Hartwig Hamer und seine Werke kennt und von 
seiner Zusammenarbeit mit Christa Wolf weiß, kann dabei durchaus von Bedeutung für die Interpretation sein.

Der Umschlag der Aufbau-Ausgabe ist durch Hamers Aquarell weniger konkret als der der Luchterhand-Ausgabe und gibt dadurch dem Leser vermutlich weniger Anhaltspunkte für den Inhalt des Textes. Gerade diese Offenheit, die unterschiedliche Interpretationen und Annäherungen an den Umschlag ermöglicht, wird von Gerhard Wolf für Hamers Aquarelle als Qualität hervorgehoben:

Das ist auch das Geheimnis der Aquarelle von Hartwig Hamer, bei dem immer weniger "Konkretes" zu sehen ist, außer Luft und Wasser und die Horizontlinie. Das geht bei ihm zunehmend in die fast abstrakte, metaphysische Bewegung. Hamer ist dabei ein ganz seßhafter, echter Mecklenburger, der mit beiden Beinen fest auf der Erde steht. (Böthig 1996, 49)

Und:

Hartwig Hamer nimmt alles Schwelgerische zurück. Wenn er auf seinem Japan-Papier die Farben zerfließen läßt, die er nur mit der Feder anskizziert hat, geht das fast bis hin in abstrakte Strukturen, wo die Elemente Wasser, Luft, Erde ineinander übergehen oder in Kontrast stehen. (ebd., 53)

Gleichzeitig kann man davon ausgehen, dass Christa Wolf ihrem ostdeutschen Publikum bestens bekannt war; eine Rezeptionssteuerung durch Umschlag und Klappentext war dadurch offenbar weniger notwendig.

Für die Umschläge der Taschenbuchserien bei dtv (1995) und Sammlung Luchterhand (2002) wurden Bilder Angela Hampels gewählt. Angela Hampels Zeichnungen wurden nicht besonders für diese Serien angefertigt, sondern von Christa und Gerhard Wolf gemeinsam mit Angela Hampel “aus einem größeren (in etwa zum Thema des

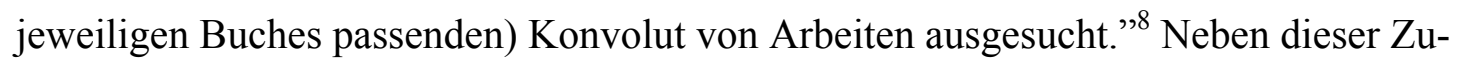
sammenarbeit war Angela Hampel sowohl an früheren als auch späteren Projekten Christa Wolfs - wie Kassandra (vgl. Böthig 1996, 46; 63ff.) und Medea (Hoch- 
geschurz 1998, 136; 159ff.) - beteiligt. Dabei ging die Idee zu einer KassandraAusstellung 1987 in Halle von Künstlern wie Angela Hampel aus:

Angela Hampel hatte ihre sehr kühnen Kassandra-Interpretationen gemacht, wo sie das KassandraThema ganz gegenwärtig auf ihre Situation bezogen auffaßte. In ihrem Zyklus waren die Kassandra's junge Punkerinnen. Sie hat ganz eigene Entwürfe aus diesem Thema entwickelt, verwandt mit ihrem Penthesilea-Zyklus, und fortgesetzt mit Undine. So entstand eine enge Freundschaft. (Böthig 1996, $63)^{9}$

Hampels Zeichnung zeigt das Gesicht einer Frau im Profil, ihr Oberkörper ist lediglich angedeutet. Die Frau trägt einen schwarzen Hut, der unsere Aufmerksamkeit auf den oberen Bildteil und das Gesicht der Frau zieht. Über dem Kopf der Frau sehen wir einen dunklen Fleck, bei dem es sich um ein Tier handeln könnte, da Hampel häufig Tiere zusammen mit Menschen - was bei ihr meist Frauen sind - abbildet.

Obwohl Hampels Zeichnung unabhängig vom Text entstand, ${ }^{10}$ kann man trotzdem davon ausgehen, dass die Bilder Hampels beim Betrachter bestimmte Assoziationen und Erwartungen an den Text auslösen und nach der Lektüre des Textes mit diesem in Verbindung gebracht werden. Wie auch Hamers Aquarell ist das Bild Angela Hampels ohne Titel wiedergegeben. Der Umschlag eines Buches mit dem Bild eines Künstlers dient hier offenbar lediglich als Blickfang und lenkt die Aufmerksamkeit des Käufers bzw. Lesers auf einen bestimmten Text, gleichzeitig weckt es beim Betrachter jedoch auch - gemeinsam mit dem Titel des Textes - Erwartungen an den Inhalt, die beim Lesen bestätigt oder enttäuscht werden können.

Vergleicht man die beiden Umschläge, ${ }^{11}$ fallen zunächst die unterschiedlichen Farben auf: Während braun in der dtv-Ausgabe dominiert, handelt es sich in der späteren Ausgabe um einen grünen Hintergrund, der im Verlagslogo und -namen wiederholt wird. Zudem ist in der späteren Ausgabe nur ein Ausschnitt des Bildes gewählt und im oberen Teil des Umschlages plaziert. Dadurch dient das Bild eher als Blickfang, durch die Wiederholung der Farbe wird jedoch auch eine Verbindung zum unteren Teil des Umschlages und zum Verlag hergestellt. In der dtv-Ausgabe fällt uns 
dagegen zuerst der Name Christa Wolf auf, der durch einen größeren Font als der Titel hervorgehoben ist. Die Dimension der Namensangabe variiert - so Genette nach dem Bekanntheitsgrad des Autors:

Diese Variationen folgen einem offenbar simplen Prinzip: Je bekannter der Autor, desto mehr breitet sich sein Name aus, aber dieser Grundsatz ist nur unter zwei Vorbehalten gültig: Erstens kann ein Autor aus außerliterarischen Gründen berühmt sein, bevor er überhaupt etwas veröffentlicht hat; zweitens betreibt der Verleger mitunter eine Werbung magischen Typs (so tun als ob, um zu erreichen, daß) und kommt dem Ruhm zuvor, indem er dessen Auswirkungen vortäuscht. (Genette 1989, 42f.)

Zwischen 1999 und 2001 erscheinen Christa Wolfs gesammelte Werke in zwölf Bänden bei Luchterhand. Die zwölfbändige Werkausgabe Christa Wolfs erschien sowohl gebunden als auch in Paperback (im Schuber), allerdings mit jeweils gleicher Umschlaggestaltung. Da sich die Bände in ihrem chronologischen Aufbau auf die Erstveröffentlichung der einzelnen Werke beziehen, enthält der 2001 erschienene Band 10 die Werke der Jahre 1989 und 1990: Sommerstück und Was bleibt. Alle Bände haben die gleiche Umschlaggestaltung - Christa Wolfs Unterschrift auf grauem Hintergrund - und haben ungefähr den gleichen Umfang. Der Schutzumschlag der gebundenen Ausgabe präsentiert im Klappentext sowohl Sommerstück und Was bleibt als auch die Autorin. Dabei ist auffallend, dass sich der Klappentext offenbar lediglich auf Ausgaben in der Sammlung Luchterhand bezieht: "Sommerstück erschien im März 1993, die Erzählung Was bleibt erschien ein Jahr danach und wurde Anlaß für den sogenannten Literaturstreit, [...].”

Jeder der zwölf Bände ist zudem mit einem Kommentar und einem Nachwort der Herausgeberin Sonja Hilzinger versehen, in dem der Leser über Entstehung, Veröffentlichung und Rezeption von Was bleibt informiert wird. Im Gegensatz zum Vorwort richten sich Genette zufolge Nachworte "nicht mehr an einen potentiellen, sondern an den tatsächlichen Leser" und gewährleisten "sicherlich eine logischere und tiefgehendere Lektüre" (Genette 1989, 228f.). Band zehn sammelt im Anhang außerdem Texte und Gedichte Christa Wolfs, die an anderer Stelle veröffentlicht wurden und durch deren Nachdruck ein literarischer Kontext zu Sommerstück und 
Was bleibt hergestellt wird. Eine Kommentierung ausgewählter Textpassagen und eine kurze Bibliographie beenden zusammen mit drei Faksimiles mit Vorarbeiten bzw. Notizen zu Sommerstück den Anhang.

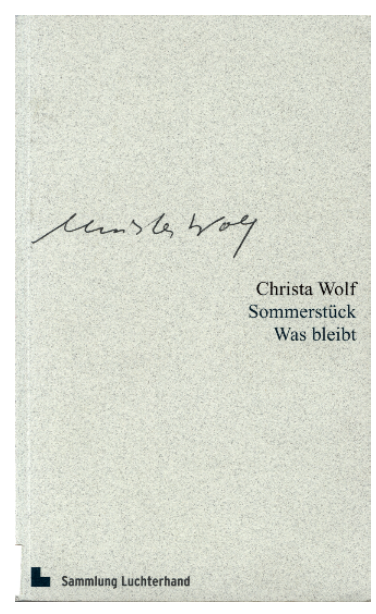

2008 erschien Sommerstück in einer neuen Serie von Christa Wolfs Texten im Suhrkamp Verlag. ${ }^{12}$ Für die Umschläge dieser Serie wurden Fotografien Christa Wolfs verwendet, die ungefähr zu dem Zeitpunkt gemacht wurden, als Christa Wolf den jeweiligen Text schrieb bzw. veröffentlichte. Die für Sommerstück gewählte Fotografie zeigt Christa Wolf vor einer flachen, weitläufigen Landschaft, offenbar in den 1980er Jahren. Eine Fotografie der Autorin lädt den Leser zur Identifikation ein; der Name der Autorin wird durch ihre Abbildung personifiziert, gleichzeitig wie die Autorin vor einer Landschaft fotografiert ist, die offenbar Ähnlichkeiten mit der im Text beschrieben hat.

Bereits der Autorenname auf der Titelseite kann als paratextuelles Element gedeutet werden, das "verschiedene [...] Merkmale der Identität des Autors" (Genette 1989, 44) angibt. Christa Wolf ist ihrem Publikum als Autorin fiktionaler Texte bekannt, der Gattungsvertrag entsteht jedoch - so Genette - "durch die Gesamtheit des Paratextes und, umfassender, durch die Beziehung zwischen Text und Paratext” (ebd., 45). Weder die ost- noch die westdeutsche Ausgabe hat eine Genrebezeichnung, die auf die Fiktionalität des Textes ausdrücklich aufmerksam machen könnte, und entsprechen dadurch eher Christa Wolfs Poetik einer subjektiven Authentizität, ${ }^{13}$ in der sich Wirkliches und Erfundenes miteinander vermischt. 
Genette weist jedoch auch darauf hin, dass die Frage inwieweit es sich um einen fiktionalen Text handelt, in vielen Fällen nur durch einen Rückgriff auf die "Ressourcen des Paratexts" beantwortet werden kann (vgl. Genette 1992, 61). Dabei müssen die Intentionen des Autors nicht unbedingt vom Empfänger als solche wahrgenommen und verstanden werden. Die von Christa Wolf proklamierte Poetik einer subjektiven Authentizität hat sicherlich dazu beigetragen, dass viele ihrer Texte eng im Verhältnis zur Wirklichkeit gelesen wurden. Gleichzeitig mögen die Existenz der Zensur und deren Eingriffe das Lesen zwischen den Zeilen und den Wunsch nach Dechiffrierung noch verstärkt haben.

So ist man im Bezug auf die Stimmentechnik in Sommerstück in der Sekundärliteratur beispielsweise darum bemüht, die einzelnen Stimmen konkreten historischen Personen zuzuordnen. ${ }^{14}$ Dies geschieht trotz des ausdrücklichen Hinweises Christa Wolfs in einem nachgestellten Satz, dass keine der Figuren mit lebenden oder toten Personen identisch ist, und dass sich die beschriebenen Episoden nicht mit tatsächlichen Vorgängen decken:

Alle Figuren in diesem Buch sind Erfindungen der Erzählerin, keine ist identisch mit einer lebenden oder toten Person. Ebensowenig decken sich beschriebene Episoden mit tatsächlichen Vorgängen. (Wolf 1989, 191)

Wie Annette Firsching bereits gezeigt hat, verweist dieser Nachtrag auf das Spiel Christa Wolfs mit den Begriffen Erfindung und Wirklichkeit (vgl. Firsching 1996, 173f.). Zwar beteuert Wolf die Fiktionalität des Textes, setzt jedoch dem Text die hintergründige Widmung “Allen Freunden jenes Sommers” voran. Außerdem unterzeichnet sie den Nachtrag mit ihren Initialen "C.W." und ist so als Autorin im Paratext anwesend. ${ }^{15}$ Dabei bleibt offen, ob es sich bei dieser Erzählerin um die Erzählerin/Ellen im Text handelt oder aber um die Autorin/Christa Wolf als Erzählerin. Da dieser Nachtrag kein Teil des fiktionalen Textes ist, sind Ich-Erzählerin und Autorin nicht automatisch identisch. Tatsächlich hat aber die Ich-Erzählerin, wie immer bei Wolf, vermutlich viele Gedanken und Gefühle der Autorin. 
Bei der Widmung “Allen Freunden jenes Sommers” handelt es sich natürlich keineswegs um eine Huldigung, "die entweder durch die Protektion feudalen Typs oder, eher bürgerlich (oder proletarisch), in Gestalt klingender Münze entlohnt wird" (Genette 1989, 117), sondern eher um eine Danksagung an Freunde, die durch ihre Anwesenheit diesen Sommer zum literarischen Gegenstand werden ließen und nicht zuletzt im Text anwesend sind.

Die unterschiedlichen Signale bezüglich der Fiktionalität von Sommerstück kommen auch in Christa Wolfs Notizen zum Arbeitsprozess zum Ausdruck. Wie auch beim Schreiben anderer Texte war die Frage nach der Erzählform unter dem Aspekt der Verfremdung bzw. Authentizität und Autobiographie wichtig. ${ }^{16}$ So notiert sie im Juni 1976:

Wie bekommt man die Subjektivität hinein, ohne allzu subjektivistisch (ich-bezogen) zu werden? Andere Erzählpersonen? Annette - Sonja? In Ich-Form? Die Leute müßten sich fast wie historische Personen von einem leuchtenden Hintergrund abheben.” (Hilzinger 2001, 315f.)

und im März 1978: "Sommerstück. - Unverstellt schreiben, die richtigen Namen, nichts erfinden. [...] Diese unheimliche Sehnsucht nach Aufrichtigkeit.” (ebd., 318)

Christa Wolfs Wunsch nach Aufrichtigkeit und Unverstelltheit muss sicherlich auch im Zusammenhang mit den Öffentlichkeitsbedingungen in der DDR und den sich daraus ergebenden Schreibbedingungen gesehen werden. Die Schwierigkeit 'ich' zu sagen, also die Frage nach individueller Identität, spielte in den Texten Christa Wolfs, aber auch in der DDR-Literatur seit den siebziger Jahren insgesamt, eine zunehmend wichtige Rolle. Nicht selten sind literarische Figuren wie die Ich-Erzählerin in Wolfs Erzählung Was bleibt in unterschiedliche Wesen gespalten, was der Doppelexistenz der meisten DDR-Bürger als öffentlicher und privater Person durchaus entspricht. Die Forderung nach Authentizität nahm deshalb in der DDR einen ausgesprochen wichtigen Platz ein, ging es doch um die Überwindung solcher schizophrenen Zustände. 
Die Widmung kann deshalb auch als eine Art Entschuldigung an die Freunde aufgefasst werden, ${ }^{17}$ zumal das Ziel einer Werkzueignung nie eindeutig ist und sich an "wenigstens zwei Adressaten" (Genette 1989, 131) wendet:

den Zueignungsadressaten, aber auch den Leser, da es sich um einen öffentlichen Akt handelt, bei dem der Leser sozusagen stets als Zeuge geladen ist. [...] Die Werkzueignung ist also immer demonstrativ, ostentativ, exhibitionistisch: Sie stellt eine intellektuelle oder private, wirkliche oder symbolische Beziehung zur Schau [...]. (ebd., 131f.)

Der Adressat der Zueignung bekommt jedoch - so Genette - ein besonderes Verhältnis zum Werk:

Erwähnt man als Auftakt oder Schlußtakt eines Werkes eine Person oder eine Sache als vorrangigen Adressaten, so wird sie zwangsläufig als eine Art idealer Inspirator einbezogen und auf die eine oder andere Weise angerufen, wie einst der Sänger die Muse anrief. "Für Soundso" enthält immer ein gewisses “Durch Soundso". Der Adressat der Zueignung ist gewissermaßen immer verantwortlich für das ihm zugeeignete Werk, dem er nolens volens ein Quentchen seiner Unterstützung und damit seiner Anteilnahme zukommen läßt. (ebd., 133. Hervorh. i. Orig.)

Wie bereits erwähnt, ist die Sekundärliteratur darum bemüht, die unterschiedlichen Stimmen in Sommerstück bestimmten Personen aus dem Freundeskreis Christa Wolfs zuzuordnen. Fotografien aus den 1970er Jahren zeigen zudem Christa und Gerhard Wolf vor ihrem Haus in Meteln zusammen mit Familie und Freunden wie Helga Schubert und Sarah Kirsch (vgl. Böthig 2004, 98f.).

Die Freunde jenes Sommers sind zudem in literarischen Zitaten im Text anwesend. So ist Sommerstück - wie vielen anderen Werken Christa Wolfs - ein literarisches Zitat vorangestellt. Sarah Kirschs Gedicht "Raubvogel süß ist die Luft” ist das letzte im Band Rückenwind, der 1976 in der DDR bei Aufbau erschien. In Rückenwind geht es - so Sarah Kirsch selbst - um den Versuch, ein Liebeserlebnis zu verarbeiten. ${ }^{18}$ Die erste und dritte Gedichtzeile werden zudem später im Text nochmals zitiert (Wolf 1989, 108) und als Zitat durch Großbuchstaben, ${ }^{19}$ jedoch ohne Nennung von Sarah 
Kirsch, kenntlich gemacht, während die zweite Zeile umschrieben den Abschnitt des Prosatextes einleitet. Durch diese Wiederholung wird eine Verbindung zwischen Peritext und Text hergestellt, gleichzeitig werden die Gedichtzeilen jedoch auch umgedeutet. So kann der Vogel als Bild für Kirschs Zentralmotiv des Fliegens gelesen werden, d.h. dem “Über-den-Dingen-Sein” (vgl. Cosentino 1981, 115). Dieses Motiv gibt dem Text eine optimistische Stimmung, kann jedoch in der Wiederholung und Umformung im Text anders gelesen werden. Wie Annette Firsching ausführt, erscheinen "die Figuren im Text alle als Gejagte, die auf die Tatsache der Jagd nur unterschiedlich reagieren" (Firsching 1996, 183).

Das Motto befindet sich auf der ersten rechten Seite, im Falle von Sommerstück noch vor der Zueignung. Letztere steht in der Ausgabe des Aufbau-Verlages auf der linken Seite nach dem Motto, gegenüber dem ersten Bild.
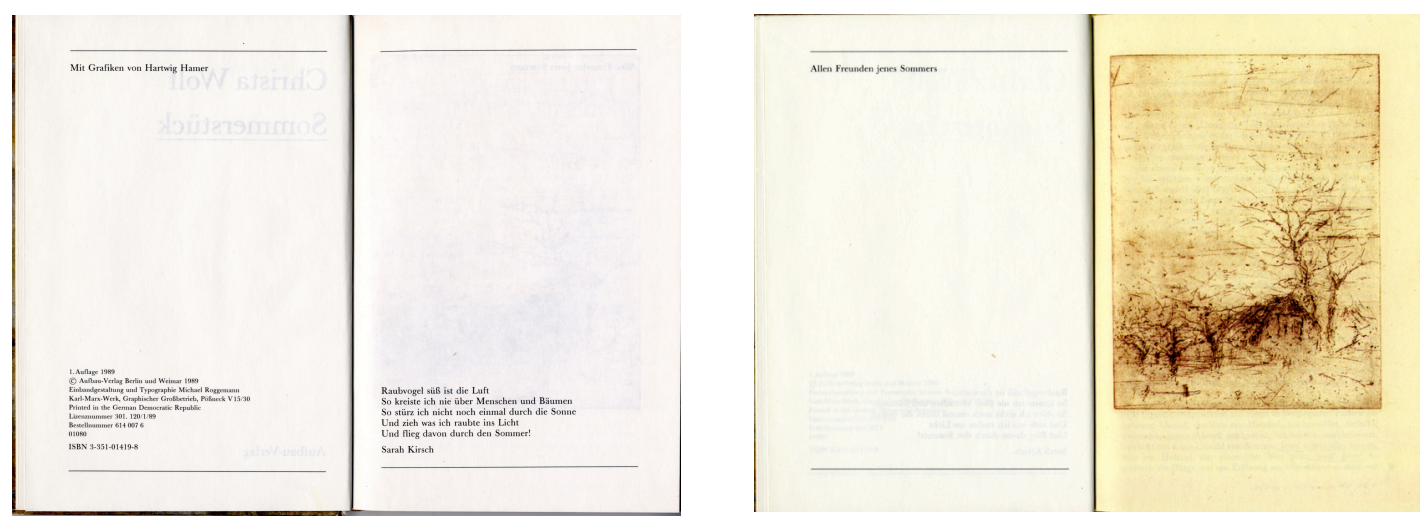

Erst auf Seite 9 beginnt der Text mit dem ersten von neunzehn Kapiteln. Sämtliche peritextuelle Elemente wie Titel, Umschlag, Motto und Widmung machen gemeinsam mit dem ersten Bild die Schwelle aus, die ein potentieller Leser übertreten muß, um in den Text zu gelangen. Sowohl Motto als auch Bild können dabei als Kommentar zum Text gelesen werden, dessen Bedeutung indirekt präzisiert und hervorgehoben wird. Die Interpretation bleibt jedoch dem Leser überlassen (vgl. Genette 1989, 152), und ist nicht zuletzt davon abhängig, inwieweit der Leser diese Elemente bewusst oder unbewusst zur Kenntnis nimmt. Während das Motto im Allgemeinen als original betrachtet werden kann und "von der ersten Ausgabe an immer enthalten" (ebd., 146) ist, entfällt die Widmung zumindest in einigen späteren Übersetzungen von Sommerstück. ${ }^{20}$ 
Inwieweit die Bilder Hartwig Hamers zu den peritextuellen Elementen gerechnet werden können, ist fraglich, da sie nicht nur den Text umgeben, sondern auch innerhalb des Textes zu finden sind. Allerdings sind die Bilder jeweils zwischen den Kapiteln plaziert und damit nicht in den Text integriert. Ein Bild am Anfang und ein Bild am Ende rahmen den Text ein. Da keine Informationen genauren Informationen zur Zusammenarbeit von Autorin und Künstler zugänglich sind, muss man annehmen dass

Word and image are not presented on the same page but refer, independently from each other, to the same event or thing in the natural world. [...] the artists have workes seperately, and the verbal-visual relation between their works exists only in the mind of the reader-beholder. (Varga 1989, 42)

Wie andere paratextuelle Elemente können Bilder - zumeist lediglich als schmückendes Element betrachtet - in späteren Ausgaben fehlen oder nach längerer Abwesenheit wieder in eine Ausgabe aufgenommen werden.

Wie bereits erwähnt finden sich Hamers Bilder lediglich in der Ausgabe des AufbauVerlages. Warum gerade Sommerstück so verhältnismäßig aufwendig gestaltet wurde, kann sicherlich mit dem Erscheinen des Textes zu Christa Wolfs 60. Geburtstag im März 1989 in Verbindung gebracht werden. Christa Wolf hat zudem von Sommerstück als ihrem persönlichsten Werk gesprochen (vgl. Wolf 1990, 149). Es ist also durchaus wahrscheinlich, dass die gewählte Kombination von Text und Bildern mit der Wichtigkeit des Textes für Christa Wolf persönlich in Verbindung steht, gleichzeitig wie der Verlag vermutlich anlässlich ihres runden Geburtstages ihren Wünschen entgegenkam.

Die Grafiken Hartwig Hamers sind im Sommerstück ohne Signatur, Jahresangabe, eventuellen Titel und Nummerierung abgebildet; lediglich auf der Kolophonseite finden wir einen Hinweis auf den Künstler: "Mit Grafiken von Hartwig Hamer". Aus dem Werkverzeichnis Hartwig Hamers erfahren wir, dass es sich bei seinen Radierungen 
durchweg um Ätzradierungen handelt. Verwendet wurden Zink-, Kupfer- und in jüngerer Zeit vermehrt Messingplatten. Gedruckt wurde auf weißem bis gelblichen Kupferdruckpapier oder -karton, seltener aus Bütten und vereinzelt auf Japanpapier. (Hamer 2003, 33)

Inwieweit die Reihenfolge der acht Grafiken und deren Anordnung zwischen den Kapiteln in Zusammenarbeit zwischen Autorin, Künstler und Verlag festgelegt wurde, geht nicht aus der Publikation hervor. Sicherlich spielten drucktechnische Faktoren und damit verbundene Kosten eine wesentliche Rolle. Da der Text überwiegt, erleben wir die Bilder als untergeordnete Elemente, die wir aber beim Lesen keineswegs übersehen. Weder ein eventueller Titel noch der Text Christa Wolfs erklären die Bilder für den Leser; durch den Text liegt jedoch die Vermutung nahe, dass die Landschaft im Text mit der auf den Bildern in Verbindung miteinander steht.

Beschäftigt man sich näher mit Hartwig Hamer, findet man im Werkverzeichnis der Radierungen die acht Motive in nummerierter Reihenfolge mit Titel (vgl. Hamer 2003, 38f.). Durch Titel wie "Haus hinter Bäumen", "Baum” oder "Einsames Gehöft" wird die Rezeption gelenkt und konkretisiert. Die im Werkverzeichnis wiedergegebene Reihenfolge der Bilder entspricht weitgehend der im Sommerstück; lediglich das vierte und fünfte Bild haben ihre Plätze getauscht.

Unsere Erwartungen an Bilder hängen von deren Umfeld ab. Im Sommerstück sind die Bilder zunächst - wie auch der Text - durch peritextuelle Elemente wie Titel, Widmung und Motto bestimmt, innerhalb des Textes sind sie von den sie umgebenen Textteilen abhängig. Die Bedeutung der Landschaft wird im Text wiederholt hervorgehoben:

Zwei Welten, das sagt man so. Aber wenn es buchstäblich zutrifft? Wenn wir lange das Gefühl nicht loswerden konnten, wir würden in ein fernes, fremdes Land eindringen, uns von ihm umschließen lassen, daß man am Ende nicht wußte, wer wen einnahm, wer wen eroberte. Aber was war es denn wirklich, und woher diese Gefühle. Die Natur, das schon, die wir zu lange kaum wahrgenommen hatten 
und die uns in unerwarteter Weise zu schaffen machte. Die Landschaft, gewiß, die uns ergriff. (Wolf $1989,69)$

Sämtliche Grafiken zeigen offenbar norddeutsche Landschaften ohne Personen, obwohl gerade das Motto "Allen Freunden jenes Sommers" eine sommerliche Landschaft mit Personen erwarten lässt. Auf den Bildern sind jedoch keine Personen anwesend und auch die Jahreszeiten lassen sich nur bedingt ablesen. Nur auf dem ersten, dem Text vorangestellten, und dem vorletzten Bild erkennen wir ein Haus. Bereits im ersten Abschnitt des ersten Kapitels erfahren wir jedoch, dass jetzt "alles zu Ende ist" und “die Häuser zerstört sind" (ebd., 9). Das vorangestellte Bild wird so zu einem vergangenen.

Indem die Bilder eine weitgehend von Menschen unberührte Landschaft darstellen, stehen sie in gewisser Weise im Gegensatz zum Text, in dem die Personen ihren Sommer in dieser Landschaft verbringen. Sogar die im Text von den Personen beobachteten Tiere fehlen:

Irene und Clemens kamen den Weg herunter. Die Männer begannen, die Vogelwelt des Weihers zu benennen. Doch, vom anderen Ufer konnte man das scheue Schwanenpaar mit seiner Brut ausmachen, im Wassergesträuch. Fünf Junge waren es, zweifelsfrei fünf. Ein Junges hielt sich so dicht hinter der Mutter, daß man es leicht übersah. (Wolf 1989, 119)

Die dargestellten Landschaften wirken dadurch abstrakter und stehen dem Leser für eigene Interpretationen offen. Sie scheinen die "heilen Flecken" (ebd., 120) zu verkörpern, die jedoch in ständiger Gefahr sind, vom Feuer zerstört zu werden: "Dann setzte eine der Feuersirenen ein, die wir in der zweiten Sommerhälfte beinahe täglich aus den umliegenden Dörfern hörten. Ununterbrochen schien es irgendwo zu brennen. Manchmal sahen wir die Rauchsäulen aufsteigen.” (ebd.)

Durch die fehlenden Titel wird dieser Interpretationsspielraum weiter vergrößert, denn Titel wie "Feld mit Weiden", "Weg nach Hause" oder "Ackerfurchen" verweisen durchaus auf die Anwesenheit von Menschen in dieser Landschaft (vgl. 
Hamer 2003, 39); Menschen, durch deren Hilfe ein Haus durchaus auch vor dem Feuer beschützt werden kann (vgl. Wolf 1989, 170ff.).

Die Kombination von Text und Bild scheint der Einsicht Christa Wolfs zu entsprechen, dass daraus etwas Neues und Anderes entsteht, etwas, "das man mit Sprache allein nicht mehr sagen kann" (ebd., 46). Die unterschiedlichen Medien ergänzen vielmehr einander, ohne dass sie notwendigerweise in Abhängigkeit voneinander geschaffen wurden:

Die Grafiken von Hamer zum Sommerstück hatten wir übrigens eigens von ihm erbeten, da wir Übereinstimmungen sahen, ohne daß er sich konkret auf den Text bezog. Es gab einen bestimmten norddeutschen Stil, der sich aus der Tradition der niederdeutschen Malerei speiste, über NiemeyerHolstein, den fast alle Mecklenburger kannten und aufsuchten. (Böthig 1996, 54)

Inwieweit Hamers Bilder aus seiner Kenntnis des Textes entstanden oder lediglich auf der Grundlage von Gesprächen mit Christa und Gerhard Wolf, geht nicht aus diesem Zitat hervor. Immerhin wird deutlich, dass es sich bei Hamers Bildern nicht unbedingt um Interpretationen zum Text handelt, so dass es schwierig ist, Aussagen zum Abhängigkeitsverhältnis zwischen Text und Bild zu machen, obwohl sie eigens für Christa Wolfs Sommerstück geschaffen wurden. ${ }^{21}$ Immerhin kann man sich vorstellen, dass andere Künstler andere Bilder zum Text schaffen können, so wie dies beispielsweise im Falle von Medea geschah (vgl. Hochgeschurz 1998). Dann würde eher zutreffen, dass "[w] hat comes first is necessarily unique; what comes after can be multiplied. One image can be the source of many texts, and one text can inspire many painters." (Varga 1989, 44)

Natürlich war Christa Wolfs Wahl nicht zufällig, denn das norddeutsche Element war offensichtlich wichtig und wird so zur Verbindung zwischen Text und Bild. ${ }^{22}$ Hamers Landschaften sind offen, der Horizont nimmt nicht selten einen großen Teil des Bildes ein. Besonders die letzten beiden Bilder ziehen den Betrachter in das Bild und damit in die Landschaft hinein und intensivieren dadurch auch die Verbindung zwischen Text und Leser. Obwohl in allen Bildern Landschaften zu erkennen sind, 
handelt es sich zum Teil doch um recht abstrakte Bilder, so dass es für den Betrachter schwierig sein kann, Gegenständliches zu erkennen, nicht zuletzt weil die Bilder in Sommerstück ohne Titel wiedergegeben werden:

Der Zeichner hält nur fest, was ihm aufzuheben wesentlich erscheint, was vor seinen Augen besteht. Sein sicheres Gefühl für diese Landschaft, die wir anzuschauen und im Wechsel der Jahreszeiten sich wandeln zu sehen nicht müde werden, kommt so auf das Blatt. Landschaft, die nichts Beschauliches oder Kleinliches kennt, die sich unserem Blick öffnet und zugleich auch wieder verschließt, mit dem elementaren Zug, den sie bis heute hat. (ebd., 203)

Wie auch Christa Wolfs Text in späteren Ausgaben ohne Hamers Bilder veröffentlicht wurde, waren Hamers Bilder als selbständige Kunstwerke ebenfalls unabhängig vom Text seinem Publikum zugänglich, sei es in Ausstellungen, Katalogen oder durch Verkauf. Hamers Radierungen in Sommerstück haben dabei auch Ähnlichkeiten zu anderen Arbeiten wie beispielsweise einer Radierung aus dem Jahre 1987 mit dem Titel "Landeinwärts" (vgl. Böthig 1996, 54) oder einer Radierung mit dem Titel "Dieses Land also", die offensichtlich direkt zu einem Text von Christa Wolf entstand und auf den Seiten des Januss-Verlages zusammen mit dem Text zu finden ist. ${ }^{23}$

In einem "Exkurs zur 'Niederdeutschen Malerei"” (Böthig 1996, 53f.) beschreibt Gerhard Wolf Hartwig Hamer als "Landschafter", der "alles Schwelgerische zurück[nimmt]" (ebd.). Gerade Hamers Art, die mecklenburgische Landschaft darzustellen, mag für Christa Wolf Anlass gewesen sein, seine Bilder für Sommerstück zu wählen:

Mich [Christa Wolf, R.S.] fasziniert immer, wenn einer nicht anders kann und überhaupt nicht zu verrücken ist. Der Hamer ist so einer, da kann man lange dran schubsen von allen Seiten, der wird immer diese Landschaften machen, auf seine Weise [...]. Dieses Absolute an ihrem Leben und an ihrer Arbeit ist es, das mich, vom Menschlichen her, aber auch vom Politischen, am meisten beeindruckt hat; abgesehen vom künstlerischen Genuß, den ich an ihren Arbeiten habe. (ebd., 53) 
Literatur

BÖTHIG, PETER (Hg.): Unsere Freunde, die Maler. Bilder, Essays, Dokumente. Berlin 1996.

-. (Hg.): Christa Wolf. Eine Biographie in Bildern und Texten. München 2004.

COSENTINO, CHRISTINE: Sarah Kirschs Dichtung in der DDR: Ein Rückblick. In:

German Studies Review 4 (1981). H. 1, S. 105-116.

FIRSCHING, ANNETTE: Kontinuität und Wandel im Werk von Christa Wolf.

Würzburg 1996 (Würzburger Beiträge zur deutschen Philologie 16).

GENETTE, GÉRARD: Paratexte. Das Buch vom Beiwerk des Buches. Mit einem

Vorwort von Harald Weinrich. Aus dem Französischen von Dieter Hornig. Frankfurt/M. 1989.

-. Fiktion und Diktion. Aus dem Französischen von Heinz Jatho. München 1992 (Bild und Text).

HAMER, HARTWIG: Meine Landschaft. Grafische Blätter. Mit einem

Werkverzeichnis der Radierungen. Hg. von Detlef Hamer. Berlin 2003.

HAYLES, KATHERINE N.: Writing Machines. Cambridge u. London 2002

(MEDIAWORK pamphlet).

HILZINGER, SONJA: Anhang. In: Christa Wolf: Kassandra. Voraussetzungen einer Erzählung. München 2000 (Werke 7). S. 399-450.

-. Anhang. In: Christa Wolf: Sommerstück. Was bleibt. München 2001 (Werke 10). S. 293-346.

HOCHGESCHURZ, MARIANNE (Hg.): Christa Wolfs Medea. Voraussetzungen zu einem Text. Mythos und Bild. Berlin 1998.

KAUFMANN, HANS: Gespräch mit Christa Wolf. In: Weimarer Beiträge. Zeitschrift für Literaturwissenschaft, Ästhetik und Kulturtheorie 20 (1974). H. 6. S. 90-112.

KUHMLEHN, THOMAS (Hg.): Angela Hampel. Eine Künstlerin in Dresden. 1982 bis 1992. Berlin 1993.

SKARE, ROSWITHA: Christa Wolfs Was bleibt. Kontext - Paratext - Text. Tromsø 2007. <http://hdl.handle.net/10037/1412>

VARGA, KIBEDI A.: Criteria for Describing Word-and-Image Relations. In: Poetics Today 10 (1989). H. 1. S. 31-53.

WOLF, CHRISTA: Sommerstück. Berlin u. Weimar 1989.

-. Sommerstück. Frankfurt/M. 1989.

-. Reden im Herbst. Berlin 1990.

-. Sommerstück. München 1995 (dtv 12003).

-. Sommerstück. Was bleibt. Herausgegeben, kommentiert und mit einem Nachwort versehen von Sonja Hilzinger. München 2001 (Werke 10).

-. Sommerstück. München 2002 (SL 2037).

-. Gestalten im Spannungsfeld. Über Angela Hampel. In: Künstler in Dresden im 20.

Jahrhundert. Literarische Porträts. Hg. von Wulf Kirsten u. Hans-Peter Lühr. Dresden 2005, S. 248-249.

-. Sommerstück. Frankfurt/M. 2008 (suhrkamp taschenbuch 3941).

Internet-Quellen

Christa Wolf liest Medea im Berliner Ensemble. 21. November 2002.

$<$ http://www.gezett.de/ lesungen/web-20021121-lit-medea-wolf-christa/index.htm $>$ Literaturbilder von Alexander Steffes $<$ http://www.ub.uni-siegen.de/expo/steffes/5$\underline{6 . h t m>}$ 
Orginalradierung von Hartwig Hamer "Dieses Land also ..." zu einem Text von Christa Wolf. $<\underline{\text { http://www.januspress.de/shop/popup_hamer_1.html }>}$

${ }^{1}$ Ich denke hier an Künstlerbücher wie Was nicht in den Tagebüchern steht (1994), Im Stein (1998) und Wüstenfahrt (1999), aber auch an die Radierungen Nuria Quevedos in der Kassandra-Ausgabe bei Reclam (1984) oder die Grafiken Hartwig Hamers in der Aufbau-Ausgabe von Sommerstück (1989). Vgl. außerdem Böthig 2004.

${ }^{2}$ Durch ihre Zusammenarbeit mit Künstlern hat Christa Wolf in den letzten Jahren wiederholt in ihren Lesungen gesprochenes bzw. gelesenes Wort mit Bild und Musik zu multimedialen Veranstaltungen verbunden. Vgl. beispielsweise die Bilder von Christa Wolfs Medea-Lesung im Berliner Ensemble vom 21. November 2002.

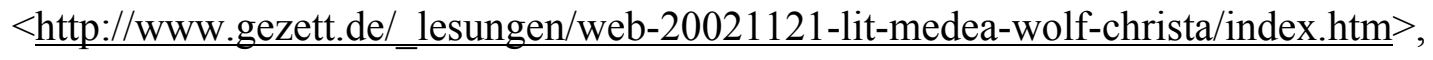
21. März 2008. Eine ähnliche Performance fand bereits im Januar 2000 statt, als Christa Wolf im Schloßtheater Rheinsberg ihren Text Im Stein las und Helge Leiberg am Overhead-Projektor malte. Vgl. Böthig 2004, 213.

${ }^{3}$ Zum Beispiel die Ausstellung Unsere Freunde, die Maler und die dazugehörige Publikation. Vgl. Böthig 1996.

${ }^{4}$ Der Deutsche Taschenbuchverlag (dtv) übernahm 1993 die "Sammlung Luchterhand" für 8 Jahre als Reihe komplett und integrierte die vorhandenen Titel in den dtv-Vertrieb. Neuauflagen dieser Titel haben dann ein "dtv-Kleid” bekommen, zunächst mit dem Hinweis "Sammlung Luchterhand", worauf man dann später in beiderseitigem Einvernehmen verzichtet hat. Vgl. elektronische Post von Helga Dick im Deutschen Taschenbuchverlag vom 15. Februar 2007.

${ }^{5}$ Die Briefe Christa Wolfs befinden sich im Verlags-Archiv des Aufbau-Verlages Berlin und Weimar in der Staatsbibliothek zu Berlin - Preussischer Kulturbesitz. Handschriftenabteilung (Mappe 2607: Depositum 38). Zitiert sind die Briefe mit Genehmigung der Staatsbibliothek in Skare 2007, 110.

${ }^{6}$ Vgl. dazu Böthig 1996, 54 u. 203. Hier sind zwei Radierungen Hamers zu

Sommerstück mit Entstehungsjahr (1988) und Größe (12,4 x 16,3) wiedergegeben. Im Buch fehlen Signatur, Jahresangabe und Titel.

${ }^{7}$ Die Umschläge sind unter Rückgriff auf das deutsche Urheberrechtsgesetz $§ 51$, Absatz 1 abgebildet.

${ }^{8}$ Elektronische Post Angela Hampels vom 23. Februar 2007. 
${ }^{9}$ An anderer Stelle spricht Christa Wolf von einer "intensive[n] Freundschaft mit Angela Hampel” (Böthig 1996, 46).

${ }^{10}$ Christa Wolf und ihr Mann haben auch in anderen Zusammenhängen dazu beigetragen, die Werke Angela Hampels einer breiteren Öffentlichkeit zugänglich zu machen. Vgl. Wolf 2005 und das bei Janus press herausgegebene Buch Angela Hampel. Eine Künstlerin in Dresden. 1982 bis 1992 (1993).

${ }^{11}$ Sucht man im Internet nach Christa Wolfs Sommerstück, findet man die unterschiedlichen Umschläge. Vgl. beispielsweise $<\underline{\text { www.amazon.de }}>$, 15. Mai 2008. ${ }^{12}$ Für den Umschlag vgl. die Internetseiten des Suhrkamp Verlages $<$ www.suhrkamp.de $>$, 15. Mai 2008.

${ }^{13}$ Christa Wolf formulierte ihre Poetik der subjektiven Authentizität erstmals 1974 in einem Gespräch mit Hans Kaufmann: "Die Suche nach einer Methode, dieser Realität schreibend gerecht zu werden, möchte ich vorläufig 'subjektive Authentizität' nennen - und ich kann nur hoffen, deutlich gemacht zu haben, daß sie die Existenz der objektiven Realität nicht nur nicht bestreitet, sondern gerade eine Bemühung darstellt, sich mit ihr produktiv auseinanderzusetzen.” (Kaufmann 1974, 95)

${ }^{14}$ Vgl. dazu Firsching 1995 und Chiarloni 1989.

${ }^{15}$ Vgl. dazu auch den Vorspann zu Kindheitsmuster: “Alle Figuren in diesem Buch sind Erfindungen der Erzählerin. Keine ist identisch mit einer lebenden oder toten Person. Ebensowenig decken sich beschriebene Episoden mit tatsächlichen Vorgängen. Wer Ähnlichkeiten zwischen einem Charakter der Erzählung und sich selbst oder ihm bekannten Menschen zu erkennen glaubt, sei auf den merkwürdigen Mangel an Eigentümlichkeit verwiesen, der dem Verhalten vieler Zeitgenossen anhaftet. Man müßte die Verhältnisse beschuldigen, weil sie Verhaltensweisen hervorbringen, die man wiedererkennt." Auch dieser Vorspann ist mit den Initialen der Autorin "C.W." unterzeichnet.

${ }^{16}$ Für Kassandra, einen Text, der ebenfalls über längere Zeit und in unterschiedlichen Fassungen entstand, experimentiert Christa Wolf in Lesungen sogar mit den unterschiedlichen Formen, um die Wirkung auf ihr Publikum zu testen. Für eine Lesung im September 1981 vermerkt sie die unterschiedlichen Reaktionen der Zuhörer in einem Erinnerungsprotokoll: "Zunächst über Ich- oder $>$ Sie $<$-Form. Ich-Form als intensiver empfunden, Identifikation mit mir stärker, Identifikationsmöglichkeiten des Lesers mit der Figur stärker. Suggestivere Form - was auch Gefahren mit sich bringt: Die 
Autorin zu stark mit der Figur - der $>$ Seherin $<-$ zu identifizieren.” (Hilzinger: 2000, 434f.)

${ }^{17}$ Vgl. Wolf 1990, 149: “Ich zögerte sehr, es zu veröffentlichen, weil es mein persönlichstes Buch ist. Ich hatte auch Hemmungen gegenüber meinen Freunden, obwohl ich sie alle in dem Buch verändert und viel dazuerfunden habe."

${ }^{18}$ Vgl. Cosentino 1981, 108. Anmerkung 13.

${ }^{19}$ In der Ausgabe von Luchterhand sind die Gedichtzeilen durch Kursivierung als Zitat kenntlich gemacht (S. 123).

${ }^{20}$ Die Widmung fehlt in der dänischen und norwegischen Übersetzung, in der finnischen Ausgabe wurde die Widmung unter das Motto gestellt.

${ }^{21}$ Vgl. Hamer 2003, 38f. Durch die technischen Angaben erfahren wir, dass es sich um Radierungen handelt, die 1988 zu Sommerstück in einer Auflage von jeweils 40 bzw. 50 Exemplaren entstanden.

${ }^{22}$ Dies schließt natürlich keineswegs aus, dass Bilder zum Text entstehen, ohne dass eine Zusammenarbeit zwischen Autorin und Künstler stattgefunden hat. Vgl. beispielsweise die Literaturbilder von Alexander Steffes $<$ http://www.ub.unisiegen.de/expo/steffes/5-6.htm>, 14. April 2008.

${ }^{23}$ Orginalradierung von Hartwig Hamer "Dieses Land also ...” zu einem Text von Christa Wolf: $<$ http://www.januspress.de/shop/popup hamer_1.html $>, 17$. März 2008. 